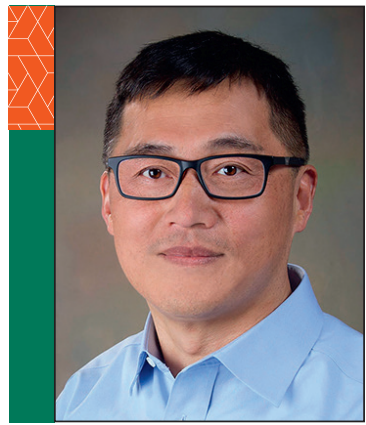

\section{Fan to be presented with the Mid-Career Researcher Award}

$\mathrm{H}$ ongyou Fan, Sandia National Laboratories and The University of New Mexico, will receive the Mid-Career Researcher Award "for outstanding contributions in nanoparticle self-assembly of functional nanomaterials and for leadership within the Materials community." The award recognizes exceptional achievements in materials research by mid-career professionals. The recipient must also demonstrate notable leadership in the materials area.

Fan is widely recognized for his pioneering work on stress-induced nanoscale assembly and fabrication technology. Stress-induced assembly uses mechanical forces to induce nanoparticles' mesophase transition for fabrication of a variety of new nanostructured metallic, semiconducting, and magnetic materials. While current nanoparticle materials synthesis methods are largely based on solution chemistry at ambient conditions, the stressinduced assembly method uses mechanical compression stresses to provide an additional processing parameter to change material structures. This enables formation of new nanomaterial configurations that impart innovative chemical and physical properties that have not been possible using chemical synthesis methods.

Recently, Fan led a major effort to extend this technology using dynamically applied electromagnetic forces to achieve high throughput fabrication with loading conditions similar to embossing and imprinting manufacturing processes but at ultrashort (nanosecond) time scales. The advantage of this approach is that nanostructured materials can be fabricated with improved performance and reduced manufacturing cost.
His team fabricated arrays of high-performance nanowire stress sensors exhibiting 10 times the density, twice the strength, and a much faster response time of $\sim 400$ nanoseconds compared to those fabricated by chemical methods.

Fan is a distinguished member of the technical staff at Sandia National Laboratories and is a National Laboratory Professor in the Department of Chemical and Biological Engineering at The University of New Mexico. He received a BS degree in chemistry from Jilin University in 1990, an MS degree in polymer science from the Chinese Academy of Sciences in 1995, and a PhD degree in chemical engineering from The University of New Mexico in 2000 . He was a postdoctoral fellow at Sandia National Laboratories, Albuquerque, N.M., before working there full-time. His research focuses on functional nanomaterials synthesis, assembly, and integration for nanoelectronic and nanophotonic applications. He is a Fellow of MRS and the American Physical Society. He has received the MRS Kavli Distinguished Lectureship Award in Nanoscience, four R\&D 100 Awards for the development of technically significant products, The University of New Mexico Outstanding Faculty Mentor Award, and the Asian American Engineer of the Year Award.

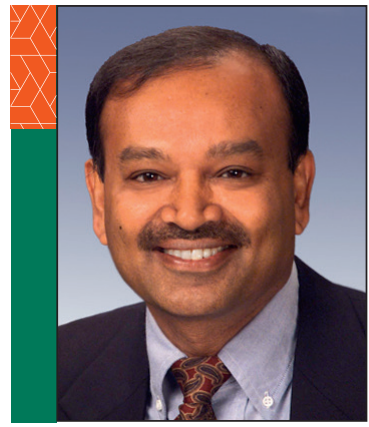

Meyyappan to receive MRS Impact Award

$\mathrm{M}$

eyya Meyyappan, NASA Ames Research Center, will receive the MRS Impact Award "for his lifelong dedication toward creating a significant and outstanding impact to understanding nanotechnology through global outreach initiatives and for unwavering mentorship."

Meyyappan arrived at NASA Ames Research Center 20 years ago and began his efforts toward education and training opportunities in nanotechnology and nanomaterials. He was one of the original four members of the Interagency Working Group on Nanotechnology (IWGN) established by the White House Office of Science and Technology Policy. The IWGN was responsible for organizing the US National Nanotechnology
Initiative. Since then, Meyyappan has helped with national nanotechnology initiatives in the United Kingdom, Israel, Republic of Korea, Thailand, Taiwan, Canada, and several other countries.

Meyyappan was instrumental in creating an IEEE website (trynano. org) in collaboration with the IEEE Educational Activities Board. This website, geared toward educating highschool students and their teachers about nanotechnology, covers topics such as what is nanotechnology and what are the applications. He has worked with community college to create nanotechnology awareness. Meyyappan also offered a NATO short course on nanomaterials for aerospace application in eight different countries with attendees from both industry and academia. 
Meyyappan is the chief scientist for Exploration Technology at NASA Ames Research Center in Moffett Field, Calif. He holds a $\mathrm{PhD}$ degree from Clarkson University, Potsdam. He has authored or co-authored more than 380 peer-reviewed journal articles, has presented more than $250 \mathrm{invited/keynote/plenary} \mathrm{talks,} \mathrm{and}$ has given more than 250 seminars at universities. He is a Fellow of MRS, IEEE, ECS, AVS, IOP, AIChE, ASME, and the National Academy of Inventors. For his contributions and leadership in nanotechnology, he has received numerous awards, including a Presidential Meritorious Award, NASA's Outstanding Leadership Medal, the IEEE Judith Resnick Award, the AIChE Nanoscale Science and Engineering Forum Award, the IEEEUSA Professional Achievement Award, the AVS Nanotechnology Recognition Award, and the AVS Plasma Prize.

For his sustained contributions to nanotechnology, Meyyappan was inducted into the Silicon Valley Engineering Council Hall of Fame in 2009. He has received an Honorary Doctorate from the University of Witwatersrand, Johannesburg, South Africa, and Concordia University, Montreal, Canada. He holds 22 nanotechnology patents, many of which have been commercialized. He also has the only nanotechnology product flown to outer space: a nano-chemical sensor monitoring air quality in the crew cabin on the International Space Station.

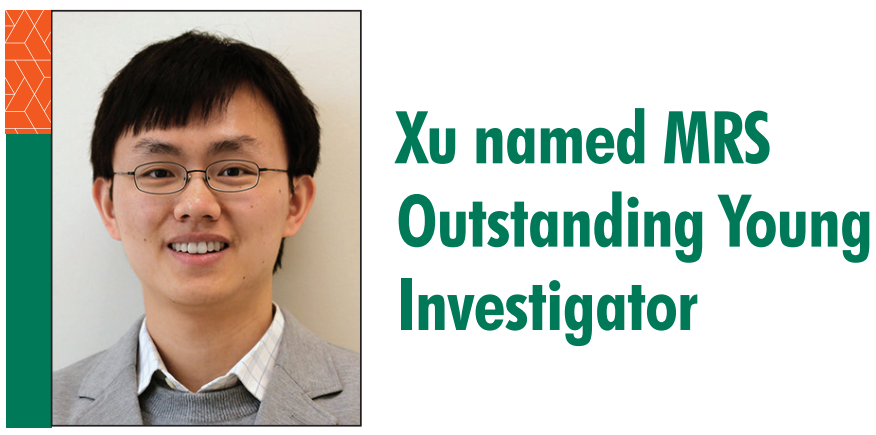

heng Xu, University of California, San $\checkmark$ Diego (UC San Diego), has received the Outstanding Young Investigator Award "for materials and device designs in biointegrated electronics and stretchable energy systems." This award recognizes outstanding interdisciplinary scientific work in materials research by a young scientist or engineer. The recipient must also show exceptional promise as a developing leader in the materials area.

$\mathrm{Xu}$ received his BS degree in chemistry and molecular engineering from Peking University in Beijing, China, and his $\mathrm{PhD}$ degree in materials science and engineering from the Georgia Institute of Technology, where he was responsible for a large fraction of the programs on growth of $\mathrm{ZnO}$ and $\mathrm{PbZrTiO}_{3}$ nanowires and their application in piezoelectric energy harvesters. In particular, he developed low-temperature solution-phase chemistries for epitaxially grown oxide nanowire arrays and schemes for integrating them into capacitor-type structures designed for converting mechanical into electrical energy. These accomplishments brought him to the Department of Materials Science and Engineering at the University of Illinois at Urbana-Champaign, where he worked as a postdoctoral research associate.

Xu's presentation on "Soft Electronics for Noninvasive Health Care-From the Skin to Below the Skin" will discuss soft electronic devices that can acquire vital signs from the human body. Combined strategies of materials design and advanced microfabrication allow the integration of a variety of components and devices on a stretchable platform, resulting in functional systems with minimal constraints on the human body. In his presentation, $\mathrm{Xu}$ will demonstrate a wearable multichannel patch that can sense a collection of signals from the human skin in a wireless mode. Additionally, integrating high-performance ultrasonic transducers on the stretchable platform adds a third dimension to the detection range of conventional soft electronics. Ultrasound waves can penetrate the skin and noninvasively capture dynamic events in deep tissues, such as blood pressure and blood flow waveforms in central arteries and veins.

$\mathrm{Xu}$ is currently an assistant professor in the Department of Nanoengineering at UC San Diego. His research group focuses on crystalline material growth for high-performance energy-harvesting devices and biointegrated electronics for health monitoring and human-machine interfaces. His research has been highlighted as "Groundbreaking Research in 2018" by Forbes and "12 innovations that will revolutionize the future of medicine" by National Geographic. He has received the MIT Technology Review 35 Innovators Under 35 Award, the NHLBI Technology Development Award, 3M Non-Tenured Faculty Award, International Union of Pure and Applied Chemistry Prize for Young Chemists, and the MRS Graduate Student Award.

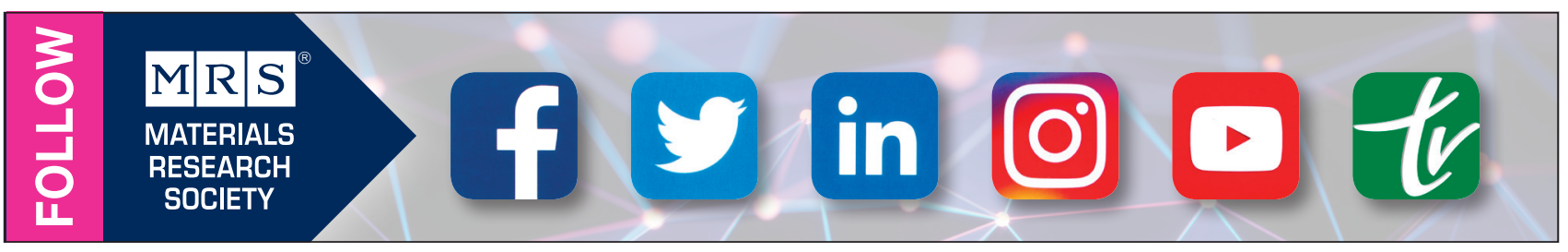

